\title{
"Efectos de una propuesta metodológica basada en el método ABP, sobre las capacidades Matemáticas de los estudiantes del Cuarto de Primaria de la I.E. 7236 "Max Uhle" de Villa El Salvador"
}

\section{"Effects of a methodological proposal based on the ABP method, on the mathematical abilities of students of the Primary Room of the I.E. 7236 "Max Uhle" from Villa El Salvador"}

\author{
Lily Marisol Pizarro Arancibia \\ Escuela Profesional de Educación / Universidad Nacional Pedro Ruiz Gallo \\ Correo-e: lili_marisol5@ hotmail.com
}

\begin{abstract}
RESUMEN
La investigación científica que se realizó es aplicada, experimental, los factores de estudio son dos: el método ABP y las capacidades matemáticas de los estudiantes del cuarto de primaria de la I.E. 7236 "Max Uhle" de Villa el Salvador. El diseño de la investigación es, experimental, cuasi experimental ya que se trabajó con dos grupos, el grupo control formado por 20 estudiantes y el grupo experimental conformado por 20 estudiantes de cuarto grado, para recolectar datos se elaboró una prueba de pretest y postest la cual evalúa las capacidades matemáticas de los estudiantes de cuarto grado de primaria de la I.E. 7236 "Max Uhle” de Villa el Salvador. El estudio planteo como objetivo determinar los efectos de la propuesta metodológica basada en el método ABP, sobre las capacidades matemáticas de los estudiantes del cuarto grado de primaria de la I.E. 7236 "Max Uhle" de Villa el Salvado. Los resultados obtenidos permiten primero evidenciar que existe una diferencia significativa entre las capacidades matemáticas de los estudiantes del cuarto grado de primaria de la I.E. 7236 "Max Uhle" de Villa el Salvador medidas antes y después de la aplicación de la propuesta metodológica basada en el método ABP esto se debe a la aplicación de las sesiones basadas en la metodología ABP y además de encontrase un valor de $p=, 000<0,05$.
\end{abstract}

Palabras clave: Metodología ABP; estudiantes; capacidades matemáticas.

\begin{abstract}
The scientific research that has been carried out is applied, experimental, the study factors are two: the ABP method and the mathematical abilities of the students of the primary room of the I.E. 7236 "Max Uhle" of Villa El Salvador. The experimental design was experimental and quasi experimental, since two groups were used: the control group of 20 students and the experimental group of 20 fourth grade students. In order to collect data, a pretest and posttest was developed. Which evaluates the mathematical abilities of the students of fourth grade of EI primary 7236 "Max Uhle" of Villa el Salvador. The objective of the study was to determine the effects of the methodological proposal based on the ABP method, on the mathematical abilities of students of the fourth grade of elementary school in the I.E. 7236 "Max Uhle" of Villa el Salvador. The obtained results allow first to show that there is a significant difference between the mathematical abilities of the students of the fourth grade of primary of the I.E. 7236 "Max Uhle" of Villa el Salvador measures before and after the application of the methodological proposal based on the $A B P$ method this is due to the application of the sessions based on the ABP methodology and besides finding a value of $p=000<0.05$.
\end{abstract}

Key words: ABP Methodology; students; mathematical abilities. 


\section{Introducción}

El área con mayor urgencia atender es Matemática, la cual se confirman con las cifras de la evaluación de rendimiento escolar que ubica al Perú en el último lugar en la matemática (UNESCO - 2002). Estos resultados constituyen una importante información acerca de las fortalezas, dificultades y necesidades del sistema educativo, que deben ser considerados para formular cualquier propuesta que apunte a una educación matemática de calidad.

El proceso de aprendizaje en nuestro país es deficiente, esto se evidencia en la cantidad de desaprobados que hay en el área de matemática es decir tienen un bajo rendimiento académico por la tanto el aprendizaje no es óptimo y esto es un problema latente y constante que se da través de los años.

Esta realidad se refleja en los resultados de la Evaluación Censal de Estudiantes (ECE) 20142015, en el 2do grado de primaria, realizada por la Unidad de Medición de la Calidad Educativa (UMC). Asimismo recogemos los resultados de la evaluación nacional 2001, realizada por la Unidad de Medición de la Calidad Educativa, UMC, donde el porcentaje de estudiantes a los que les gusta la matemática decrece al pasar del nivel de Educación Primaria al nivel de Educación Secundaria. Una probable explicación es que, con el tiempo, los estudiantes enfrentan mayores dificultades en la medida en que existe mayor exigencia y complejidad en el desarrollo de capacidades para enfrentar nuevos retos. En lo que respecta al currículo, las capacidades, para cuyo desarrollo se ofrecieron menos oportunidades de aprendizaje, son las referidas al desarrollo de la resolución de problemas.

Por otro lado la ECE se aplica al final del año escolar, entre los meses de noviembre y diciembre. Se trata de una evaluación estandarizada a gran escala que produce información comparable acerca de los desempeños de los estudiantes de todo el país. El objetivo de la ECE es identificar el nivel de apropiación de la noción de número y sus operaciones en el campo aditivo en que se encuentran los estudiantes evaluados de segundo grado de primaria, así como su habilidad de utilizar esta comprensión en formas flexibles para hacer juicios matemáticos y desarrollar estrategias útiles para resolver problemas. Como observamos en la tabla 1 de los Resultados Nacionales, donde el incremento en el nivel satisfactorio, del 2014 al 2015, solo se hizo en un $0,7 \%$.

Tabla 1. Matemática: niveles de logro 2014 - 2015. Resultados Nacionales

\begin{tabular}{|c|c|c|c|c|c|}
\hline \multicolumn{4}{|c|}{ Matemática: niveles de logro 2014 - 2015} & \multicolumn{2}{|c|}{ UMC 9 mew } \\
\hline \multicolumn{6}{|c|}{ Resultados nacionales } \\
\hline \multirow{2}{*}{\multicolumn{2}{|c|}{ Resultados }} & ECE 2014 & ECE 2015 & \multirow{2}{*}{ Diferencia } & \\
\hline & & $\mathbf{4}$ & \% & & \\
\hline \multirow{3}{*}{ Por niveles de logro } & Satisfactorio & 25,9 & 26,6 & 0,7 & \\
\hline & En proceso & 35,3 & 42,3 & $7,0^{*}$ & \\
\hline & En inicio & 38.7 & 31.0 & $-7,7^{*}$ & \\
\hline
\end{tabular}

Nota: Fuente: Tomado de Unidad de Medición de la Calidad (UMC) 2016. Resultados de ECE, 2do grado de primaria, 2015.

Los estudiantes de Educación Primaria tienen dificultades para traducir y expresar matemáticamente las condiciones propuestas en problemas de enunciado verbal, aplicar estrategias de solución para obtener la respuesta y justificarla con argumentos matemáticos válidos. Según MED-DINEBR (2006) menciona que los estudios internacionales como el de PISA, realizado con estudiantes de 15 años, los peruanos han mostrado un bajo nivel de desempeño en la resolución de problemas, incluso en aquellas tareas en las que la formulación matemática está explícita; así también, en la resolución de un problema rutinario en el que sólo se exige un paso para su solución. (p. 12)

Por lo tanto, las actividades que tradicionalmente se realiza no les permiten tener relación existente entre la matemática y el mejoramiento de la calidad de su vida personal y social; así mismo 
no comprenden qué desarrollo, de las ciencias sociales y el avance tecnológico actual han sido posibles, en gran parte, debido al uso instrumental de la matemática. Esta situación se observa con mayor incidencia en las Instituciones Educativas ubicadas en entornos con niveles de desarrollo socioeconómico más bajos, sobre todo en aquellas ubicadas en zonas urbanas marginales.

Villanueva y Pillman (2006) mencionan que según los estudios realizados en Villa El Salvador en el $73.7 \%$ de los colegios, el curso con mayor cantidad de desaprobados es matemática, y le sigue Comunicación con $57.5 \%$. El porcentaje de estudiantes desaprobados en ambos cursos es del 44\%, y la mayor cantidad de desaprobados en estos cursos es en el nivel de primaria. (p. 21)

En general, la calidad educativa es deficiente, las condiciones en las que se educa son precarias, y la escuela en VES se ha aislado de la vida de la comunidad, perdiendo gradualmente el rol que tuvo en los primeros tiempos de la historia de VES y que debería recuperarlo. En cuanto a la infraestructura educativa se observa que los colegios secundarios en los primeros sectores del distrito concentran la mayor cantidad y mejor calidad de espacios recreativos, laboratorios, talleres, bibliotecas, auditorios y equipos de cómputo, evidenciándose una gran diferencia con los colegios secundarios de la periferia, en este caso los colegios del Parque Metropolitano de Villa El Salvador que carecen de servicios básicos de agua y desagüe.

Así mismo Villanueva y Pillman (2006) manifiestan que el magisterio en Villa El Salvador está conformado básicamente por docentes jóvenes (36 años en promedio), con un $63 \%$ de presencia femenina. El $87 \%$ de los profesores que trabajan en las escuelas públicas poseen título profesional. La necesidad de capacitación que ellos reconocen, está en función de temas relacionados con el fortalecimiento personal (autoestima), los aprendizajes para el ejercicio ciudadano, el trabajo en equipo y el trabajo con los padres de familia, entre otros. (p. 22)

Con respecto a las necesidades de nuestra Institución Educativa N ${ }^{\circ} 7236$ "Max Uhle", ello se manifiesta en el bajo nivel de aprendizaje en los estudiantes en lo que va del año 2016 y anteriores y este análisis gracias a que tenemos documentos de evaluación de los trimestres, y es en donde coexisten muchos factores, destacando entre ellos, como factor que incide con mayor fuerza, en el bajo nivel de aprendizaje en el área de Matemática, la aplicación de métodos para su enseñanza y aprendizaje.

Muy a pesar de la intensa difusión estatal de los principios básicos de la pedagogía activa desde hace más de dos décadas por parte del Ministerio de Educación, queda claro para todo aquel académico que tenga contacto con la Educación Básica regular en el Perú, que para la asunción de la pedagogía activa dentro de la práctica docente es necesario un proceso mucho más profundo, que no se limite a la inoculación de conceptos que luego se convertirían en un discurso vacío e inasible por parte del magisterio nacional. Los conceptos manejados durante la década de los 70 fueron luego tildados de tradicionalistas por los nuevos regímenes, propugnando la propia monserga como el mensaje innovador de la pedagogía activa. El maestro queda así a merced de mensajes confusos sin poder mejorar su práctica pedagógica.

El método ABP, se inscribe dentro de las propuestas de la pedagogía activa, por lo que el presente estudio se propone la indagación respecto a los resultados de la implementación de una propuesta que pretende cubrir el vacío que encuentra el docente peruano para la mejora de su práctica pedagógica.

En el trabajo de investigación de Rayego, Sanz y García (2006), titulado Un ensayo de la ABP en la enseñanza de las matemáticas. Se propone facilitar el aprendizaje y reducir el nivel de abstracción, con la concreción de cada uno de los grandes apartados de que consta el programa de la asignatura, mediante la resolución de problemas en un entorno colaborativo, utilizando como material auxiliar software libre y auto evaluación, así como tratar de ver si esta metodología aplicada de forma adecuada, podría complementar si se perfecciona, las clásicas pruebas presénciales, constituyéndose en un sistema de evaluación continua complementario.

Otro trabajo estudiado es el de González (2006), titulado: Propuesta didáctica para la aplicación de la enseñanza basada en problemas a la formación Semipresencial en la disciplina de Geometría. República de Cuba. Instituto Superior Pedagógico "Enrique José Varona", Facultad de Ciencias Exactas. La Habana - Cuba. $\mathrm{La}$ investigadora en correspondencia con lo anterior se propone como objetivo: diseñar una propuesta didáctica, basada en problemas, para contribuir al perfeccionamiento del proceso de enseñanza-aprendizaje de la disciplina Geometría en la formación semipresencial de profesores de Ciencias Exactas. Llega a la conclusión de que su aplicación puede contribuir a conferir un carácter desarrollador al proceso de enseñanza aprendizaje. Entre las ramas de la Matemática, la Geometría presenta condiciones especialmente favorables para la aplicación de esta tendencia, pues en ella predominan los procedimientos heurísticos (p. 25). 
Contribuye a fundamentar el estudio, igualmente el trabajo de Morales y Ortega (2008), titulado: Aprendiendo los conceptos de sistema de referencia, parábola y línea recta mediante el uso del método de aprendizaje basado en problemas aplicado a la solución de una situación experimental en física. Departamento de Física, Facultad de Ciencias Físicas y Matemáticas, Universidad de Concepción. Chile. Los investigadores mencionan que en el primer curso de matemáticas elementales muchos estudiantes reprueban, aun cuando las materias (en cuanto a contenidos) son las que debieron estudiarse en Enseñanza Media. Este hecho los ha motivado para intentar mejorar el rendimiento académico de los estudiantes, así como incentivar el razonamiento crítico y capacidad de análisis en aquellas personas que han reprobado una o dos veces los primeros cursos de matemáticas. La metodología de la propuesta fue la de aprendizaje basado en la resolución de problemas, donde diez estudiantes se inscribieron en el curso; dos profesores, un docente del área de matemáticas y un docente del área de Física; Los investigadores llegaron a la conclusión que indudablemente el método ABP funciona bien, y en el sentido de que estudiantes con desventajas académicas obtienen un aprendizaje bastante más significativo que usando el método tradicional de enseñanza. (p. 49).

Con fundamento en lo expuesto, se formula la siguiente pregunta de investigación: ¿Cuáles son los efectos de la propuesta metodológica basada en el método ABP, sobre las capacidades matemáticas de los estudiantes del cuarto grado de primaria de la I.E. 7236 "Max Uhle" de Villa el Salvador? Los objetivos del estudio son los siguientes: (1) Determinar los efectos de la propuesta metodológica basada en el método ABP, sobre la capacidad de los estudiantes para traducir situaciones. (2) Determinar los efectos de la propuesta metodológica basada en el método ABP, sobre la capacidad de los estudiantes para comunicar ideas matemáticas. (3) Determinar los efectos de la propuesta metodológica basada en el método ABP, sobre la capacidad de los estudiantes para usar estrategias en la resolución de problemas matemáticos. (4) Determinar los efectos de la propuesta metodológica basada en el método ABP, sobre la capacidad de los estudiantes para argumentar generando ideas matemáticas.

La hipótesis que guía el estudio es que existe una diferencia significativa entre las capacidades matemáticas de los estudiantes del cuarto grado de primaria de la I.E. 7236 "Max Uhle" de Villa el Salvador medidas antes y después de la aplicación de la propuesta metodológica basada en el método ABP.

\section{Materiales y métodos}

\section{Objeto del estudio}

La unidad de análisis de la presente investigación es un estudiante de la I.E. 7236 "Max Uhle" de Villa el Salvador.

\section{Población y muestra}

El estudio se ha realizado con 40 estudiantes pertenecientes a las secciones A y B del cuarto grado de primaria de la I. E. $\mathrm{N}^{\circ} 7236$ Max Uhle del distrito de Villa El Salvador UGEL No 01. Cada sección está compuesta por 20 estudiantes.

\section{Instrumentos de recolección de datos}

Lista de cotejo denominada Cuestionario sobre capacidades matemáticas, construido por la autora de este estudio.

\section{Método}

\section{Técnicas}

Observación.

\section{Diseño de estudio}

En el marco de la clasificación de diseños de Hernández, Fernández y Baptista (2015), el diseño se define como correlacional, determinándose la asociación entre las variables mediante la prueba Chi cuadrado.

\section{Procedimiento:}

- Se coordinó con La Institución Educativa y se obtiene los permisos para la toma de encuestas a los docentes.

- Se aplicó los la Propuesta metodológica.

- Se tabuló, analizó y discutió los datos.

- Se redactó las conclusiones y sugerencias en base a la discusión de resultados

- Se comunicaron las conclusiones del trabajo de investigación 


\section{Resultados}

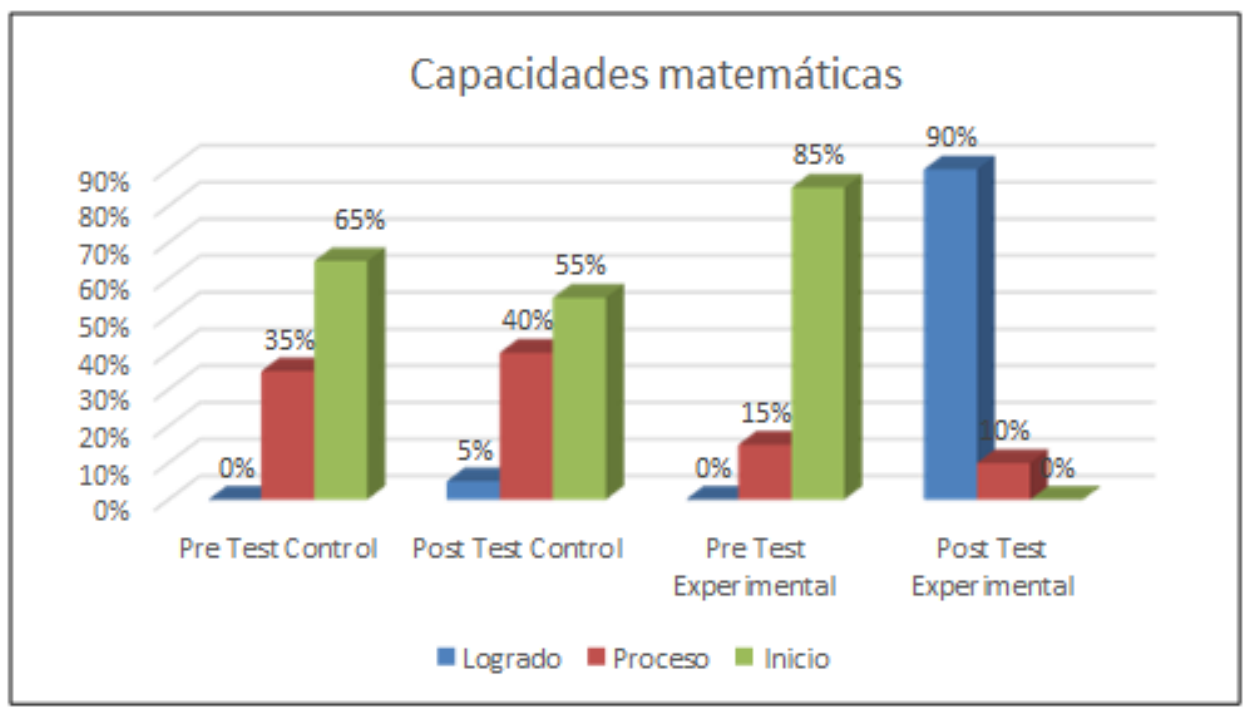

Figura 1. Se observa que en el pre test con respecto al grupo control el $35 \%$ de los evaluados se encuentran en el nivel inicio, el $65 \%$ en el nivel de proceso; mientras que en el grupo experimental se da que el $85 \%$ de los evaluados se encuentran en el nivel inicio, el $15 \%$ en el nivel proceso. En el pos test se observa que en el grupo control, el $55 \%$ está en un nivel inicio, el $40 \%$ está en un nivel proceso y el $5 \%$ en un nivel logrado; mientras que en el grupo experimental el $90 \%$ está en un nivel logrado y el $10 \%$ en un nivel inicio en cuanto al desarrollo de las capacidades matemáticas.

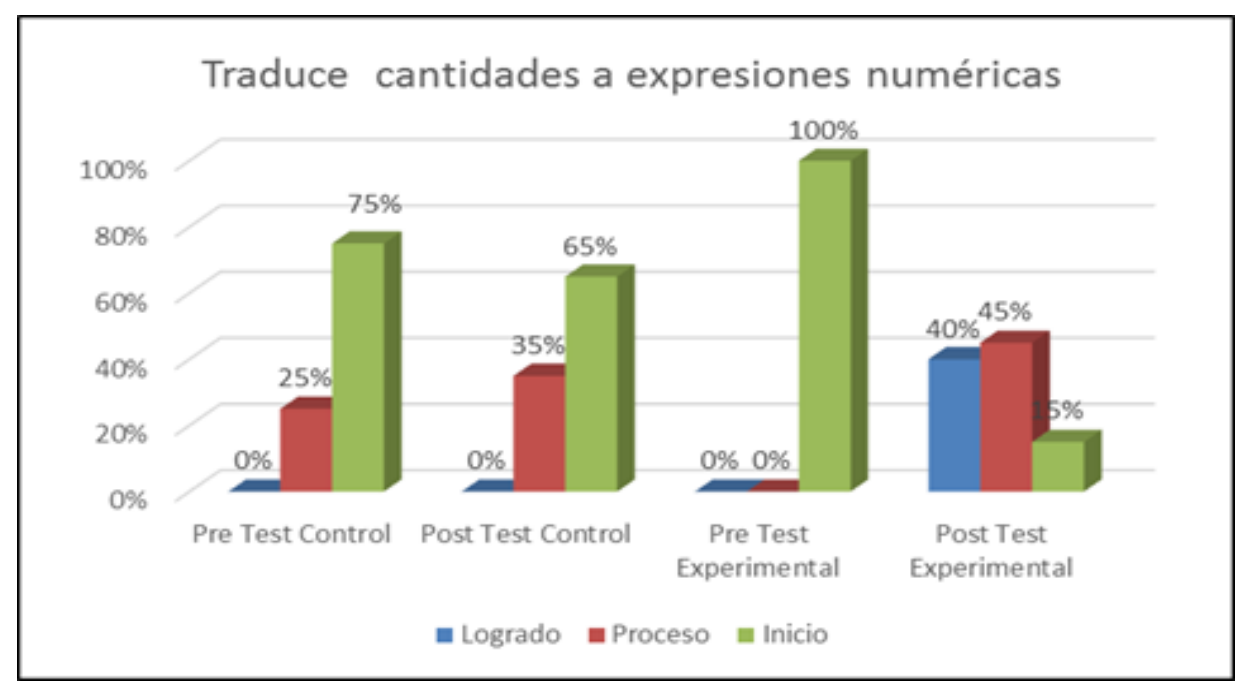

Figura 2. En el pre test con respecto al grupo control el $75 \%$ de los evaluados se encuentran en el nivel inicio, el $25 \%$ en el nivel proceso; mientras que en el grupo experimental se da que el $100 \%$ de los evaluados se encuentran en el nivel inicio. En el pos test se observa que en el grupo control, el $65 \%$ está en un nivel inicio, y el $35 \%$ en un nivel de proceso el mientras que en el grupo experimental el $40 \%$ está en un nivel logrado, el $14 \%$ en un nivel proceso y el $15 \%$ en un nivel de inicio en cuanto a la dimensión traduce cantidades a expresiones numéricas. 


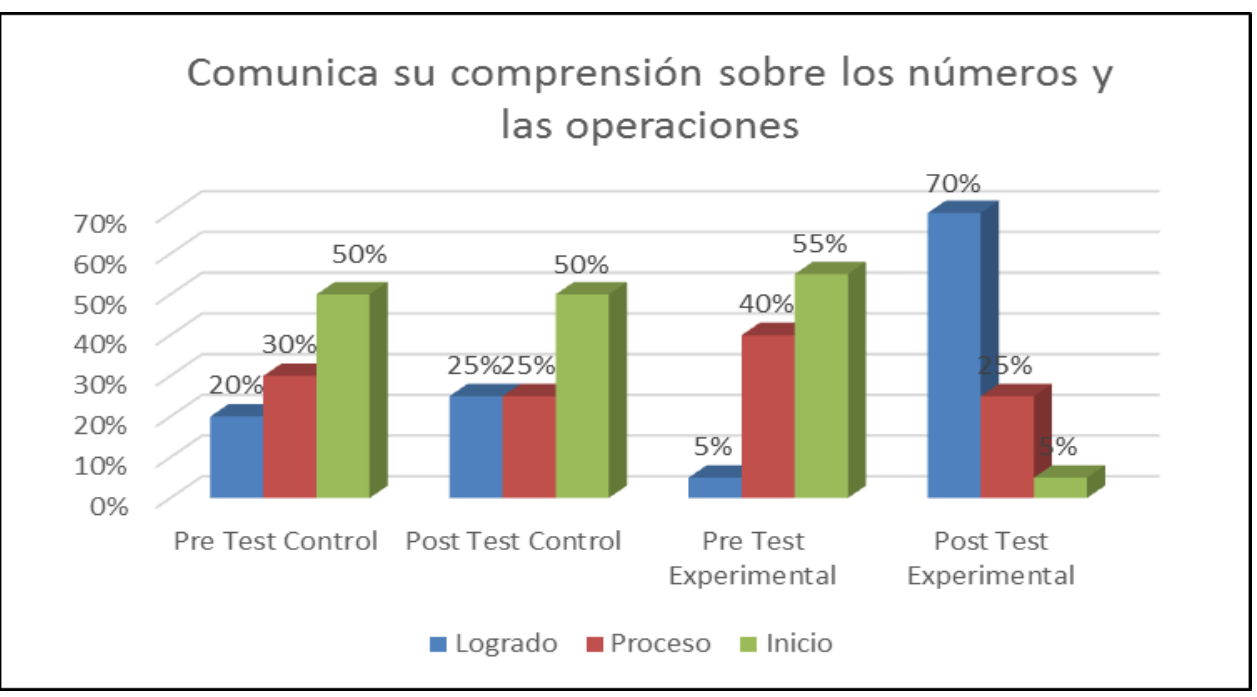

Figura 3. En el pre test con respecto al grupo control el $50 \%$ de los evaluados se encuentran en el nivel de inicio, el $30 \%$ en el nivel proceso y el $20 \%$ en el nivel logrado; mientras que en el grupo experimental se da que el 55\% de los evaluados se encuentran en el nivel de inicio, el $40 \%$ en el nivel de proceso y el 5\% en un nivel logrado. En el pos test se observa que en el grupo control, el $50 \%$ está en un nivel inicio, el 255 en un nivel de proceso y el 255 en n nivel logrado; mientras que en el grupo experimental el $70 \%$ está en un nivel logrado, el $25 \%$ en un nivel de proceso y el $5 \%$ en un nivel de inicio en cuanto a la dimensión comunica su comprensión sobre los números y las operaciones.

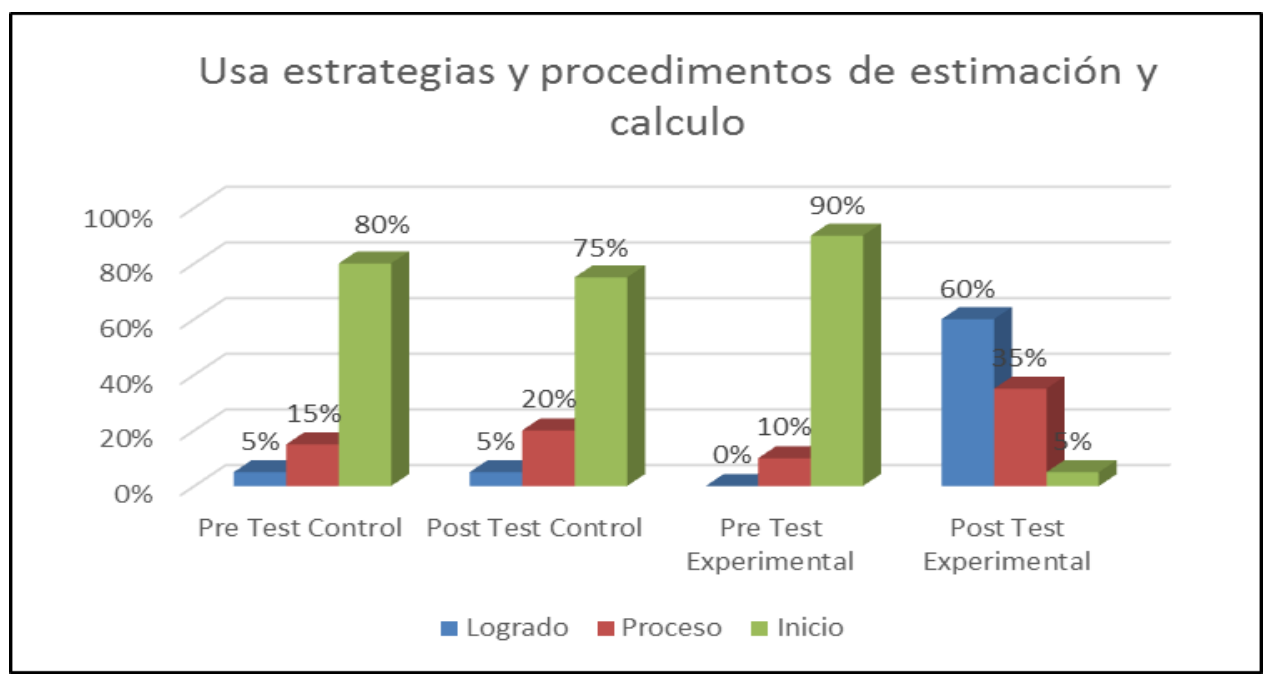

Figura 4. En el pre test con respecto al grupo control el $80 \%$ de los evaluados se encuentran en el nivel de inicio, el $15 \%$ en el nivel de proceso y el 55 en un nivel de logrado; mientras que en el grupo experimental se da que el $90 \%$ de los evaluados se encuentran en el nivel de inicio, el $10 \%$ en el nivel de proceso. En el pos test se observa que en el grupo control, el $75 \%$ está en un nivel de inicio, el $20 \%$ en un nivel de proceso y el $5 \%$ en un nivel logrado; mientras que en el grupo experimental el $60 \%$ está en un nivel logrado, el $35 \%$ en un nivel de proceso y el $5 \%$ en un nivel de inicio en cuanto a la dimensión estimación y calculo. 


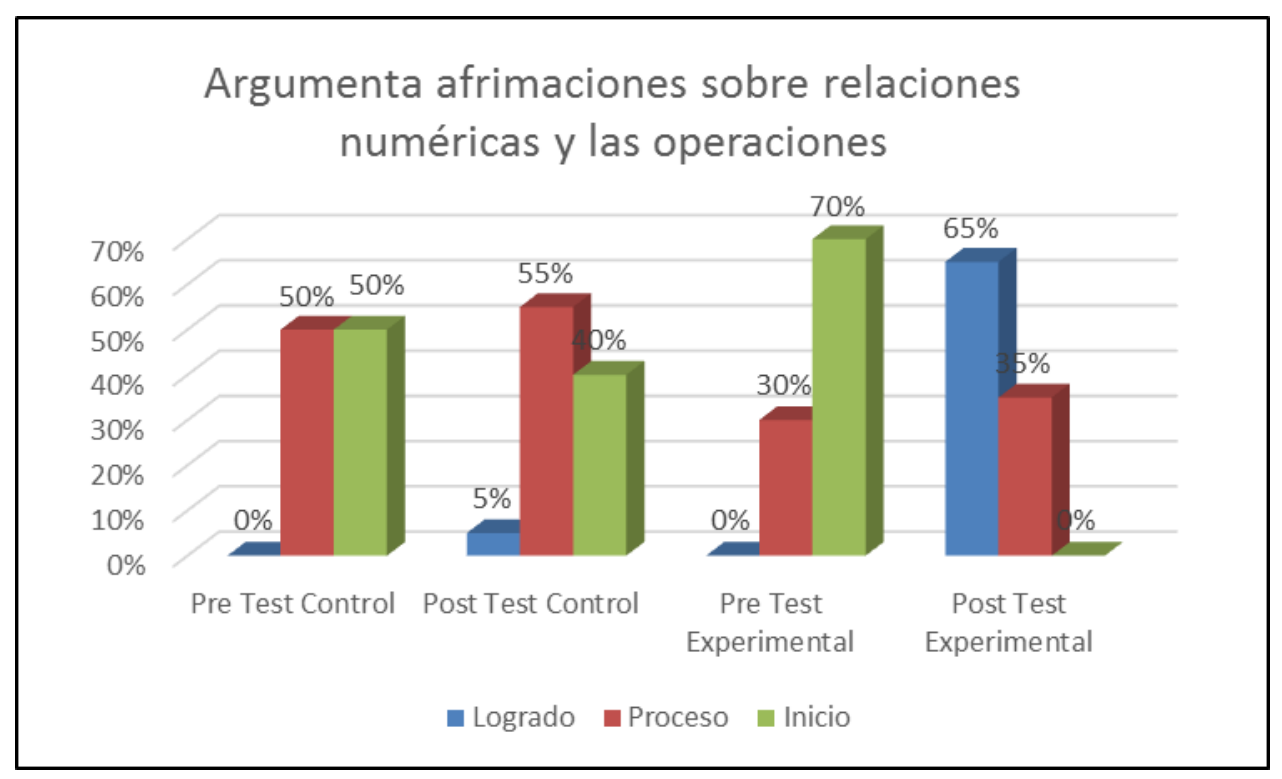

Figura 5. En el pre test con respecto al grupo control el $50 \%$ de los evaluados se encuentran en el nivel de inicio, el $50 \%$ en el nivel de proceso; mientras que en el grupo experimental se da que el $70 \%$ de los evaluados se encuentran en el nivel de inicio, el $30 \%$ en el nivel de proceso. En el pos test se observa que en el grupo control, el $40 \%$ está en un nivel de inicio, el $55 \%$ en un nivel de proceso y el $2 \%$ en un nivel logrado; mientras que en el grupo experimental el 65\% está en un nivel logrado, el $35 \%$ en un nivel de proceso en cuanto a la dimensión argumenta afirmaciones sobre relaciones numéricas y las operaciones.

Tabla 2. Prueba de la hipótesis general. Capacidades matemáticas pre y post test.

\begin{tabular}{lrr}
\hline & pretest & postest \\
\hline U de Mann-Whitney & 60,000 &, 000 \\
W de Wilcoxon & 138,000 & 78,000 \\
Z &,- 714 & $-4,239$ \\
Sig. asintótica (bilateral) &, 475 &, 000 \\
\hline
\end{tabular}

En la prueba $U$ de Mann Whitney el Valor $P(0,000)<0,05$ se rechaza la hipótesis nula y se acepta la hipótesis alterna además de evidenciar que en el pretest y postest existen diferencias significativas por lo cual se decide aceptar que existe una diferencia significativa entre las capacidades matemáticas de los estudiantes del cuarto grado de primaria de la I.E. 7236 "Max Uhle" de Villa el Salvador medidas antes y después de la aplicación de la propuesta metodológica basada en el método $A B P$. 
Tabla 3. Capacidad para traducir situaciones

\begin{tabular}{lrr}
\hline & D1PRETS & D1POSTS \\
\hline U de Mann-Whitney & 155,000 & 64,500 \\
W de Wilcoxon & 365,000 & 274,500 \\
Z & $-1,395$ & $-3,851$ \\
Sig. asintótica (bilateral) &, 163 &, 000 \\
Significación exacta [2*(sig. unilateral)] &, $231^{\mathrm{b}}$ &, $000^{\mathrm{b}}$ \\
\hline
\end{tabular}

Nota: (a). Variable de agrupación: GRUPO. (b). No corregido para empates.

En la prueba $U$ de Mann Whitney el Valor $P(0,000)<0$, se evidencia que en el pretest y postest existen diferencias significativas por lo cual se decide aceptar que existe una diferencia significativa la capacidad de los estudiantes para traducir situaciones, medida antes y después de la aplicación de la propuesta metodológica.

Tabla 4. Capacidad para comunicar ideas matemáticas.

\begin{tabular}{lrr}
\hline & D2PRET & D2POST \\
\hline U de Mann-Whitney & 177,000 & 87,500 \\
W de Wilcoxon & 387,000 & 297,500 \\
Z &,- 691 & $-3,287$ \\
Sig. asintótica (bilateral) &, 490 &, 001 \\
Significación exacta [2*(sig. unilateral)] &, $547^{\mathrm{b}}$ &, $002^{\mathrm{b}}$ \\
\hline
\end{tabular}

Nota: (a). Variable de agrupación: GRUPO. (b). No corregido para empates.

En la prueba $U$ de Mann Whitney el Valor $P(0,000)<0,05$ evidencia que en el pretest y postest existen diferencias significativas por lo cual se decide aceptar que existe una diferencia significativa entre la capacidad de los estudiantes para comunicar ideas matemáticas, medida antes y después de la aplicación de la propuesta metodológica.

Tabla 5. Capacidad para usar estrategias en la resolución de problemas matemáticos

\begin{tabular}{lrr}
\hline & D2PRET & D2POST \\
\hline U de Mann-Whitney & 155,000 & 36,000 \\
W de Wilcoxon & 365,000 & 246,000 \\
Z & $-1,336$ & $-4,603$ \\
Sig. asintótica (bilateral) &, 182 &, 000 \\
Significación exacta [2*(sig. unilateral)] &, $231^{\mathrm{b}}$ &, $000^{\mathrm{b}}$ \\
\hline
\end{tabular}

Nota: (a). Variable de agrupación: GRUPO. (b). No corregido para empates.

En la prueba $U$ de Mann Whitney el Valor $P(0,000)<0,05$ evidencia que en el pretest y postest existen diferencias significativas por lo cual se decide aceptar que existe una diferencia significativa entre la capacidad de los estudiantes para usar estrategias en la resolución de problemas matemáticos, medida antes y después de la aplicación de la propuesta metodológica. 
Tabla 6. Capacidad para argumentar generando ideas matemáticas

\begin{tabular}{lrr}
\hline & D2PRET & D2POST \\
\hline U de Mann-Whitney & 160,000 & 52,000 \\
W de Wilcoxon & 370,000 & 262,000 \\
Z & $-1,275$ & $-4,321$ \\
Sig. asintótica (bilateral) &, 202 &, 000 \\
Significación exacta [2*(sig. unilateral)] &, $289^{\mathrm{b}}$ &, $000^{\mathrm{b}}$ \\
\hline
\end{tabular}

Nota: (a). Variable de agrupación: GRUPO. (b). No corregido para empates.

En la prueba $U$ de Mann Whitney el Valor $P(0,000)<0,05$ evidencia que en el pretest y postest existen diferencias significativas por lo cual se decide aceptar que existe una diferencia significativa entre la capacidad de los estudiantes para argumentar generando ideas matemáticas, medida antes y después de la aplicación de la propuesta metodológica.

\section{Discusión}

El proceso de los datos entorno a la hipótesis general permito aceptar que Existe una diferencia significativa entre las capacidades matemáticas de los estudiantes del cuarto grado de primaria de la I.E. 7236 "Max Uhle" de Villa el Salvador medidas antes y después de la aplicación de la propuesta metodológica basada en el método ABP, al encontrar el valor de $\mathrm{p}=, 000<0,05$, al respecto la investigación Rayego, Sanz y García (2006), en su estudio titulado "Un ensayo de la ABP en la enseñanza de las matemáticas" Dpto. Economía Aplicada Cuantitativa Facultad de Ciencias Económicas y Empresariales UNED, con el fin de facilitar el aprendizaje y reducir el nivel de abstracción, con la concreción de cada uno de los grandes apartados de que consta el programa de la asignatura, mediante la resolución de problemas en un entorno colaborativo el cual logro mejoras significativas.

El trabajo de Morales y Ortega (2008), titulado: "Aprendiendo los conceptos de sistema de referencia, parábola y línea recta mediante el uso del método de aprendizaje basado en problemas aplicado a la solución de una situación experimental en física" Departamento de Física, Facultad de Ciencias Físicas y Matemáticas, Universidad de Concepción. Chile. Estos trabajos analizados me permiten iniciar nuestra base teórica y llevar a cabo la aplicación del método ABP en nuestra muestra seleccionada.

En cuanto a la hipótesis especifica llegó a evidenciar que Existe una diferencia significativa entre la dimensión la capacidad de los estudiantes para traducir situaciones, la capacidad de los estudiantes para comunicar ideas matemáticas, la capacidad de los estudiantes para usar estrategias en la resolución de problemas matemáticos y la capacidad de los estudiantes para argumentar generando ideas matemáticas, medida antes y después de la aplicación de la propuesta metodológica, al respecto González (2006), en su estudio titulado: "Propuesta didáctica para la aplicación de la enseñanza basada en problemas a la formación Semipresencial en la disciplina de Geometría". República de Cuba. Instituto Superior Pedagógico "Enrique José Varona”, Facultad de Ciencias Exactas. La Habana - Cuba. La investigadora asume como objeto de Estudio: El proceso de enseñanza-aprendizaje de la disciplina Geometría en la formación de profesores de Ciencias Exactas en condiciones semipresenciales. Y considera como Campo de Acción: el proceso de enseñanza-aprendizaje de la disciplina Geometría, basado en problemas, en la formación de profesores en condiciones semipresenciales. También menciona que brinda una propuesta didáctica que, sustentada en la aplicación de la enseñanza basada en problemas en condiciones semipresenciales, utiliza las tecnologías de la información y las comunicaciones para favorecer la participación activa de los estudiantes en la elaboración de los conocimientos y el establecimiento de métodos de trabajo matemático, a partir de la reflexión metacognitiva de los procedimientos heurísticos aplicados.

\section{Conclusión}

1. Se evidenció en cuanto al objetivo general que existe una diferencia significativa entre las capacidades matemáticas de los estudiantes del cuarto grado de primaria de la I.E. 7236 "Max Uhle" de Villa el Salvador medidas antes y después de la aplicación de la propuesta metodológica basada en el método ABP. 
2. En cuanto a la hipótesis específica primera se encontró que existe una diferencia significativa la capacidad de los estudiantes para traducir situaciones, medida antes y después de la aplicación de la propuesta metodológica.

3. Con respecto a la hipótesis específica dos se determinó que existe una diferencia significativa entre la capacidad de los estudiantes para comunicar ideas matemáticas, medida antes y después de la aplicación de la propuesta metodológica.

4. Con respecto a la hipótesis específica tercera se determinó que existe una diferencia significativa entre la capacidad de los estudiantes para usar estrategias en la resolución de problemas matemáticos, medida antes y después de la aplicación de la propuesta metodológica.

5. Con respecto a la hipótesis específica cuarta se determinó que existe una diferencia significativa entre la capacidad de los estudiantes para argumentar generando ideas matemáticas, medida antes y después de la aplicación de la propuesta metodológica.

\section{Referencias bibliográficas}

[1] Arellano, T. (2006) La Educación Matemática en el Perú. Perú: Sociedad Peruana de Educación Matemática.

[2] |Delgado, K. (2007) Educacion participativa: el metodo del trabajo en grupos. 2da. Ed., Colombia: Cooperativa Editorial Magisterio.

[3] Díaz, M., Izquierdo, W. y Mendoza, M. (2006) Orientaciones para el Trabajo Pedagógico de Matemática. Perú: Fimart S.A.C

[4] Escribano, A. y Del Valle, A. (2008) El aprendizaje basado en problemas: una propuesta metodológica en educación superior. Narcea Ediciones

[5] García, L. (2006) Psicología Cognitiva. Lima: PROLIC. CREPREDIM. UNMSM.

[6] González, M. (2006) Propuesta didáctica para la aplicación de la enseñanza basada en problemas a la formación Semipresencial en la disciplina de Geometría. Instituto Superior Pedagógico "Enrique José Varona", Facultad de Ciencias Exactas. La Habana - Cuba.

[7] Hernández, R. Fernández, C. y Baptista, P. (2006) Metodología del Investigación. Editoriales McGRAW-HIL, México.
[8] Ledesma, L. y Liza, P. (2008) Teorias y estrategias de aprendizaje. LIMA:UCV

[9] León, C. (2006) Guía para el desarrollo del pensamiento crítico. Perú: MED Fimart S.A.C

[10] Matus, R. y Guzmán, J. (2009). Uso del aprendizaje basado en problemas en un curso de matemáticas. México: CETYS. Tijuana

[11] MED-DINEBR. (2006) Propuesta Pedagógica para el desarrollo de las Capacidades Matemáticas: Matemática para la vida. Perú.

[12] Ministerio de Educación (2008) Diseño Curricular Nacional. Lima Perú. 2008

[13] Ministerio de Educación (2015) Rutas de Aprendizaje Versión 2015. Lima

[14] Monsalve, S. y Smith, C. (2004) Psicología General. Perú- Lambayeque: FACHSE

[15] Morales, J. y Ortega, M. (2008) Aprendiendo los conceptos de sistema de referencia, parábola y línea recta mediante el uso del método de aprendizaje basado en problemas aplicado a la solución de una situación experimental en física. Chile: Departamento de Física, Facultad de Ciencias Físicas y Matemáticas, Universidad de Concepción.

[16] Morales, P. y Landa V. (2004) Aprendizaje basado en problemas. Theoria, Vol. 13: Lima PUCP.

[17] Rayego, P., Sanz, B. y García, C. (2006) Un ensayo de la $A B P$ en la enseñanza de las matemáticas Dpto. Economía Aplicada Cuantitativa Facultad de Ciencias Económicas y Empresariales UNED. España.

[18] Santillán, F. (2006) El aprendizaje basado en problemas como propuesta educativa para las disciplinas económicas y sociales apoyadas en el b-learning. Centro Universitario de Los Valles, Universidad de Guadalajara, México.

[19] Serrano, J. (2004) El desarrollo del pensamiento lógico-matemático. Conferencia de Apertura del $1^{\circ}$ Congreso Mundial de Matemáticas en Universidad de Murcia.

[20] Villanueva, S. y Pillman, A. (2006) Diagnóstico local participativo del consumo de drogas en el distrito de Villa El Salvador. Perú: CICAD-OEA-AE. 
